\title{
MicroRNA-155-5p Overexpression in Peripheral Blood Mononuclear Cells of Chronic Lymphocytic Leukemia Patients Is a Novel, Independent Molecular Biomarker of Poor Prognosis
}

\author{
Sotirios G. Papageorgiou, ${ }^{1}$ Christos K. Kontos, ${ }^{2}$ Marios A. Diamantopoulos, ${ }^{2}$ \\ Anthi Bouchla, ${ }^{1}$ Eirini Glezou, ${ }^{1}$ Efthymia Bazani, ${ }^{1}$ Vasiliki Pappa, ${ }^{1}$ and Andreas Scorilas ${ }^{2}$ \\ ${ }^{1}$ Second Department of Internal Medicine and Research Unit, University General Hospital "Attikon”, 1 Rimini St., Haidari, \\ 12462 Athens, Greece \\ ${ }^{2}$ Department of Biochemistry and Molecular Biology, National and Kapodistrian University of Athens, Panepistimiopolis, \\ 15701 Athens, Greece
}

Correspondence should be addressed to Christos K. Kontos; chkontos@biol.uoa.gr

Received 26 September 2017; Accepted 20 November 2017; Published 31 December 2017

Academic Editor: Alvaro González

Copyright (C) 2017 Sotirios G. Papageorgiou et al. This is an open access article distributed under the Creative Commons Attribution License, which permits unrestricted use, distribution, and reproduction in any medium, provided the original work is properly cited.

\begin{abstract}
MicroRNA-155-5p (miR-155-5p) is a proinflammatory, oncogenic miRNA, involved in various physiological processes, including hematopoiesis, immunity, inflammation, and cell lineage differentiation. It regulates important transcription factors, such as E2F2, hypoxia-inducible factor 1 (HIF1), and FOXO3. Recently, the dysregulation of miR-155-5p expression has been linked to chronic lymphocytic leukemia (CLL) pathogenesis. In this research study, we investigated the potential diagnostic and prognostic value of miR-155-5p in CLL. To achieve our goal, we isolated total RNA from peripheral blood mononuclear cells (PBMCs) collected from 88 CLL patients and 36 nonleukemic blood donors and performed polyadenylation of total RNA and reverse transcription. Next, we quantified miR-155-5p levels using an in-house-developed real-time quantitative PCR method, before proceeding to extensive biostatistical analysis. Thus, it appears that miR-155-5p is significantly overexpressed in PBMCs of CLL patients and can distinguish them from nonleukemic population. Kaplan-Meier OS analysis and bootstrap univariate Cox regression showed that high miR-155-5p expression predicts inferior OS for CLL patients $(p<0.001)$. Interestingly, miR-155-5p overexpression retains its unfavorable prognostic role in CLL patients stratified according to established prognostic factors [CD38 expression and mutational status of the immunoglobulin heavy chain variable region $(I G H V)]$. Thus, miR-155-5p appears as a promising, independent molecular biomarker of unfavorable prognosis in CLL.
\end{abstract}

\section{Introduction}

Chronic lymphocytic leukemia (CLL), the most common type of leukemia in adults, remains incurable, although patients' survival has been extended with discovery and implementation of new treatment options $[1,2]$. The features of CLL comprise the progressive accumulation of small mature $\mathrm{CD}^{+} \mathrm{B}$ lymphocytes in the peripheral blood, lymphoid organs, and bone marrow [3]. The clinical course of its hematological malignancy is remarkably heterogeneous among patients [2]; most of them are elderly people. The average age at the time of CLL diagnosis is about 71 years, while it is rarely encountered in people under the age of 40 and is very uncommon in children [1].

Currently, two systems of disease classification are routinely used to predict the prognosis of CLL patients, namely, the Rai [4] and the Binet staging [5] systems. Though they may be useful, both staging systems do not predict therapy failure. Moreover, they are unable to discriminate between patients with probably evolving disease and those achieving stable disease for a long life period [6]. The prognosis of CLL patients is still based on clinicopathological finding; however, extensive research in the field during the last decade has unleashed the prospect 
of adding new prognostic biomarkers for CLL in the clinical routine [7], such as cytogenetic markers, mutational status of the immunoglobulin heavy chain variable region $(I G H V)$, CD38 expression, and zeta-chain-associated protein kinase $70 \mathrm{kDa}$ (ZAP70) expression in leukemic B cells $[8,9]$. In this regard, partial deletion of chromosome 13 (not combined with other karyotype abnormalities), low percentage of ZAP70-positive and/or CD38-positive leukemic B cells (20\% and/or $30 \%$, resp.), and a mutated IGHV locus are indicative of favorable prognosis in CLL [10].

MicroRNAs (miRNAs) are small single-stranded noncoding RNA molecules that epigenetically regulate gene expression, mostly through binding to the $3^{\prime}$ untranslated region ( $3^{\prime}$-UTR) of targeted messenger RNA (mRNA) molecules and, hence, mediating translational repression, usually along with mRNA degradation too [11]. It is estimated that the human genome can produce more than a thousand of different miRNAs, which regulate approximately $60 \%$ of protein-coding genes, thereby controlling the activity and function of key signaling pathways and cellular processes such as cell proliferation, apoptosis, cell differentiation, and response to hypoxia [12]. In fact, miRNAs can function as either oncogenes or tumor suppressors, and some of the$\mathrm{m}$-including miR-15a, miR-16, miR-181b, and members of the miR-17/92 cluster-play pivotal roles in CLL onset and progression [13]. From a clinical perspective, particular miRNAs can also serve as biomarkers for CLL onset [14].

miR-155-5p is a proinflammatory, oncogenic miRNA, highly expressed in activated $\mathrm{B}$ and $\mathrm{T}$ cells as well as in macrophages. It is involved in various physiological processes, including hematopoiesis, immunity, inflammation, and cell lineage differentiation. Targets of miR-155-5p include several cytosolic and nuclear proteins that control the development of regulatory T cells [15]. For instance, miR-155-5p targets E2F2 transcription factor to enhance the angiogenic capability of induced endothelial cells [16]. Another target of miR155-5p is hypoxia-inducible factor 1 alpha subunit (HIF1A), namely, the regulatory $\alpha$-subunit of the heterodimeric HIF1 transcription factor [17]. HIF1 is a major effector of hypoxia, as it regulates many genes involved in cellular proliferation, migration, energy metabolism, angiogenesis, and apoptosis $[18,19]$. Moreover, HIF1 is the transcription factor primarily responsible for miR-155-5p induction in hypoxia [17]. This oncomiR shows upregulated expression levels in several malignant solid tumors [20-22]. Aberrant expression of miR-155-5p has also been observed in diffuse large B cell lymphoma, acute myeloid leukemia, and CLL [23, 24]. Moreover, transgenic mice with B cells overexpressing miR-155$5 p$ are very likely to develop B cell lymphomas [25].

The aforementioned evidence prompted us to assess the prognostic and diagnostic potential of miR-155-5p in CLL and to evaluate its putative clinical application as a molecular biomarker in this hematological malignancy. To achieve this goal, we developed an accurate and cost-effective quantitative real-time PCR (qPCR) methodology based on the SYBR ${ }^{\circledR}$ Green chemistry, for the relative quantification of miR-155$5 p$ levels in leukemic B cells of CLL patients and PBMCs of normal blood donors.

\section{Materials and Methods}

2.1. Patients and Control Group. This original research study included 88 patients who had previously been diagnosed with CLL at the Second Department of Internal Medicine and Research Unit in University General Hospital "Attikon" (Athens, Greece) as well as 36 normal controls. None of the patients had received any treatment during the last six months before blood sample collection. Peripheral blood mononuclear cells (PBMCs) were positively selected after centrifugation on a Ficoll-Hypaque gradient. Most PBMCs isolated from patients' blood samples were leukemic B cells, as confirmed by cell immunophenotyping. The clinical stage of CLL was determined based on the Binet and Rai staging systems. Patients with Rai stage 0 CLL were considered to have a low risk, patients of stage I and II were considered to have an intermediate risk, and stage III and IV patients were classified in the high-risk group.

This research study was approved by the institutional Ethics Committee of the University General Hospital "Attikon" (Athens, Greece) and conducted in respect to the ethical standards of the Helsinki Declaration of 1964, as revised in 1983. A written informed consent was also signed from each participant of the study.

2.2. Cell Line Culture. The human histiocytic lymphoma cell line U-937 was propagated appropriately in Dulbecco's modified Eagle's medium, adjusted to contain $10 \%$ fetal bovine serum, $100 \mathrm{kU} / \mathrm{L}$ penicillin, $0.1 \mathrm{~g} / \mathrm{L}$ streptomycin, and $2 \mathrm{mM} / \mathrm{L}$ glutamine. Cells were seeded at a concentration of $0.5 \times 10^{5}$ cells $/ \mathrm{mL}$ and incubated for $48 \mathrm{~h}$ at $37^{\circ} \mathrm{C}$, in a humidified atmosphere containing $5 \% \mathrm{CO}_{2}$, before being collected for further use.

2.3. Cell Immunophenotyping. Immunophenotyping of CLL cells was performed with a routine panel of evaluated monoclonal antibodies (Supplementary Materials and Methods is available here). Based on a previously established threshold [9], cases with more than $30 \%$ of CD38-expressing cells were characterized as positive for this marker, as previously described [26].

2.4. Nucleic Acid Extraction, RNA Polyadenylation, and Reverse Transcription of Poly (A) RNA. DNA and total RNA were extracted from isolated PBMCs and U-937 cells using the TRI Reagent ${ }^{\circledR}$ (Molecular Research Center Inc., Cincinnati, $\mathrm{OH})$. The concentration and purity of purified total RNA were assessed with a BioSpec-nano Micro-volume UV-Vis Spectrophotometer (Shimadzu, Kyoto, Japan), and the purified total RNA was stored in deep freeze until further use. Next, total RNA polyadenylation and first-strand cDNA synthesis using an oligo-dT adapter primer were performed, as previously described in detail [27].

2.5. Determination of the IGHV Mutational Status. PCR amplification and sequencing-based analysis of IGHV$I G H D-I G H J$ gene rearrangements were performed as described in Supplementary Materials and Methods. IGHV genomic sequences were considered to be mutated when 
the homology with the closest germ line counterpart was less than $98 \%$, as previously described [26].

2.6. RT-qPCR. The developed RT-qPCR methodology exploited the SYBR Green chemistry and was applied in a 7500 Fast Real-Time PCR System (Applied Biosystems, Foster City, CA, USA). Based on the annotated sequences of mature miR-155-5p and SNORD48 (also known as $R N U 48$ or $U 48$ ), we designed two specific forward primers which are used along with a common reverse primer to generate two amplicons (68 and $105 \mathrm{bp}$, resp.). Primer sequences and the detailed RT-qPCR protocol are presented in Supplementary Materials and Methods.

The relative miR-155-5p expression was determined using the comparative $\mathrm{C}_{\mathrm{T}}\left(2^{-\Delta \Delta \mathrm{Ct}}\right)$ method $[28,29]$, and its prerequisites were checked in a validation experiment. The normalized miR-155-5p expression of each sample was calculated as the ratio of miR-155-5p molecules to SNORD48 molecules, divided by the same ratio that had been previously calculated for the calibrator (U-937 cells), and was measured in relative quantification units (RQU).

2.7. Biostatistical Analysis. The distributions of miR-155-5p expression values in B-CLL patients and normal individuals were not Gaussian; hence, the nonparametric Mann-Whitney $U$ test was used to check whether there was a statistically significant difference between the distributions of the two cohorts. We also assessed the diagnostic potential of miR155-5p expression by receiver operating characteristic (ROC) analysis. Thus, a ROC curve was built by plotting sensitivity versus 1 - specificity, and the respective area under the curve (AUC) was analyzed by the Hanley and McNeil method. Logistic regression was also carried out for the prediction of B-CLL occurrence.

For the determination of the best cut-off value and subsequent categorization of patients into miR-155-5p-positive and miR-155-5p-negative groups, we used the X-tile software [30]. This cut-off point was 1.84 RQU, equal to the 77 th percentile. Associations between miR-155-5p expression status and other categorical clinicopathological variables were assessed with the chi-square test or the Fisher exact test, where appropriate.

Kaplan-Meier overall survival (OS) analysis was performed, and differences between OS curves were evaluated using the Mantel-Cox (log-rank) test. Moreover, Cox proportional hazard regression models were built for the assessment of the association between the prognostic markers and the relative risk of succumbing to CLL. The reliability of the developed Cox proportional hazard regression models was strengthened by carrying out bootstrapping in Cox regression analysis, using a number of 2000 samples as well as bias-corrected and accelerated (BCa) 95\% confidence intervals. The multivariate Cox regression models were adjusted for the CD38 expression status, presence of $I G H V$ mutations, and either clinical stage (Binet or Rai) or risk group. Stratification of B-CLL patients participating in the current study into distinct subgroups with similar prognosis based on established prognostic factors followed by KaplanMeier OS analysis was also performed. The level of statistical significance was set at a probability value of less than $0.050(p<0.050)$, regarding all statistical tests.

\section{Results}

3.1. Biological and Clinical Characteristics of CLL Patients. The group of patients consisted of 62 men and 26 women, with a median age of 70 years (range: $50-90$ years) at the time of diagnosis. Using the Binet staging classification, 50 CLL patients $(56.8 \%)$ were at stage $\mathrm{A}, 16(18.2 \%)$ at stage $\mathrm{B}$, and $22(25.0 \%)$ at stage C. Positive CD38 expression was encountered in $15(17.0 \%)$ cases, while $73(83.0 \%)$ patients had CD38-negative leukemic B cells. In addition, the early apoptosis index of clonal B cells varied between 0.01 and 54.8 (median value: 4.7 ), and $I G H V$ mutations were detected in 45 (51.1\%) CLL cases, whereas 43 (48.9\%) patients had wild-type $I G H V$. Biological and clinical features of the CLL patients are presented in Table 1.

3.2. miR-155-5p Expression in PBMCs of CLL Patients and Normal Individuals. In leukemic cells, miR-155-5p expression ranged from 0.081 to 3.99 RQU with a mean \pm SE of $1.22 \pm 0.11$, while in PBMCs of normal samples, the levels of this miRNA varied between 0.11 and 1.06 RQU with a mean \pm SE of $0.33 \pm 0.030$ (Figure $1(\mathrm{a})$ ); this difference was statistically significant $(p<0.001)$. On the other hand, miR155-5p expression was not associated with CLL patients' age, white blood cell count, lymphocyte count, CD38 expression, or early apoptosis index.

Univariate logistic regression analysis revealed that high miR-155-5p expression could predict CLL occurrence (crude odds ratio $=28.37,95 \% \mathrm{CI}=5.20-154.82, p<0.001$ ). More interestingly, ROC analysis showed that miR-155-5p expression can clearly distinguish CLL patients from normal controls (area under the curve (AUC) $=0.81,95 \%$ confidence interval (95\% CI) $=0.74-0.88, p<0.001)$, as also illustrated in Figure 1(b).

3.3. Independent Unfavorable Prognostic Value of miR-155-5p Overexpression in CLL Patients. Based on the optimal prognostic cut-off point that was determined for miR-155-5p expression values, as described in detail in Materials and Methods, 68 (77.2\%) CLL cases were categorized as miR155-5p-negative and 20 (22.7\%) as miR-155-5p-positive.

Follow-up information was available for all patients, 28 (31.8\%) of whom died during the accrual period due to causes related to CLL. The estimated median OS was 73 months (95\% CI=45-101 months). Kaplan-Meier analysis revealed significantly reduced OS for miR-155-5p-positive CLL patients in comparison with miR-155-5p-negative patients $(p<0.001)$ (Figure 2). In agreement with these results, univariate Cox regression analysis uncovered a significant $(p=0.001)$, quite 4 -fold higher risk of death for CLL patients exhibiting miR-155-5p positivity (Table 2). Bootstrap Cox regression models highlighted the robustness of the conclusion that miR-155-5p overexpression is a significant unfavorable prognosticator in CLL $(p<0.001)$ (Table 2).

Most importantly, the unfavorable prognostic significance of miR-155-5p overexpression was shown to be 
TABLE 1: Biological and clinicopathological features of CLL patients.

\begin{tabular}{|c|c|}
\hline Total number of patients & 88 \\
\hline \multirow[t]{2}{*}{ Patients' sex (male/female) } & $62 / 26$ \\
\hline & Median (range) \\
\hline Age (years) & $70(50-90)$ \\
\hline OS (months) & $42(4-120)$ \\
\hline $\begin{array}{l}\text { White blood cells } \\
\left(\times 10^{6} / \mathrm{mL}\right)\end{array}$ & $32,250(8500-96,900)$ \\
\hline Lymphocytes $\left(\times 10^{6} / \mathrm{mL}\right)$ & $23,050(5300-69,430)$ \\
\hline $\begin{array}{l}\text { CD38 expression } \\
\text { (mean optical intensity of staining) }\end{array}$ & $7.7(0.10-59.2)$ \\
\hline \multirow[t]{2}{*}{$\begin{array}{l}\text { Early apoptosis index } \\
\text { ( } 55 / 88 \text { patients) }\end{array}$} & $4.7(0.01-54.8)$ \\
\hline & Number of patients (\%) \\
\hline \multicolumn{2}{|l|}{ Binet stage } \\
\hline A & $50(56.8 \%)$ \\
\hline $\mathrm{B}$ & $16(18.2 \%)$ \\
\hline C & $22(25.0 \%)$ \\
\hline \multicolumn{2}{|l|}{ Rai stage } \\
\hline 0 & $16(18.2 \%)$ \\
\hline I & $27(30.7 \%)$ \\
\hline II & $24(27.3 \%)$ \\
\hline III & $4(4.5 \%)$ \\
\hline IV & $17(19.3 \%)$ \\
\hline \multicolumn{2}{|l|}{ Risk group } \\
\hline Low & $16(18.2 \%)$ \\
\hline Intermediate & $51(58.0 \%)$ \\
\hline High & $21(23.9 \%)$ \\
\hline \multicolumn{2}{|l|}{ Serum LDH concentration } \\
\hline Normal & $60(68.2 \%)$ \\
\hline Elevated & $28(31.8 \%)$ \\
\hline \multicolumn{2}{|l|}{ CD38 expression status } \\
\hline Negative & $73(83.0 \%)$ \\
\hline Positive & $15(17.0 \%)$ \\
\hline \multicolumn{2}{|l|}{$I G H V$ mutational status } \\
\hline Mutated & $45(51.1 \%)$ \\
\hline Unmutated & $43(48.9 \%)$ \\
\hline
\end{tabular}

CLL: chronic lymphocytic leukemia; OS: overall survival; LDH: lactate dehydrogenase; IGHV: immunoglobulin heavy chain variable region.

independent of $\mathrm{CD} 38$ expression, unmutated $I G H V$, and the clinical stage (Binet or Rai stage) or risk group, as demonstrated in the multivariate Cox regression analysis and illustrated by the respective forest plots (Figure 3).

3.4. Unfavorable Prognostic Value of miR-155-5p Overexpression in Distinct Subgroups of CLL Patients, Stratified According to Established Prognostic Factors. As the prognosis of patients with negative CD38 expression and/or unmutated $I G H V$ genomic sequences is substantially different from the prognosis of patients showing positive CD38 expression and/or mutated IGHV, Kaplan-Meier OS analysis was performed in substantially different prognostic subgroups of CLL patients, stratified according to each one of these established prognostic factors, in order to evaluate the potential additional prognostic value of miR-155-5p expression. As clearly illustrated in Figure 4(a), miR-155-5p positivity predicts an unfavorable outcome also among CLL patients with negative CD38 expression $(p=$ 0.010). Similarly, CLL patients with unmutated IGHV sequence combined with strong miR-155-5p expression had significantly lower OS probabilities than those with unmutated $I G H V$ and negative miR-155-5p expression status $(p=0.007)$ (Figure $4(\mathrm{~b}))$.

\section{Discussion}

miRNAs play a major role in almost every normal cellular process in animals, including normal hematopoiesis. Dysregulation of their expression levels contributes significantly to the development and/or progression of many human diseases, including cancer and leukemias [31]. Several miRNAs have been suggested as promising therapeutic targets in the battle against leukemia, as restoration of their normal expression in leukemic cells can dramatically alter the expression levels of key proteins that are heavily involved in leukemia pathobiology [32]. Moreover, some of these small noncoding RNAs have prognostic value in particular hematological malignancies [32].

miR-155-5p constitutes an important miRNA in lymphoid differentiation. High levels of miR-155-5p are present in activated $\mathrm{B}$ and $\mathrm{T}$ cells and in activated monocytes [33]. miR-155-5p tunes finely germinal center reaction and $\mathrm{T}$ helper cell differentiation by affecting cytokine production [34]. Furthermore, miR-155-5p controls the function of lymphocytes and dendritic cells and is critical for normal immune function [35]. Of note, miR-155-5p targets forkhead box 3 (FOXO3) [36], a transcription factor acting downstream of the PTEN/PI3K/AKT pathway and being indispensable for self-renewal of hematopoietic stem cells [37]. miR-155-5p expression is upregulated in AML patients with FLT3-ITD mutations [38-41]. On the other hand, sustained expression of miR-155-5p is associated with enhanced myeloid proliferation [42]. Thus, miR-155-5p seems to play a significant role in the pathogenesis of a particular subgroup of AML patients [41]. Very recently, the dysregulation of the miR-155-5p/miR-150 network has been linked to CLL pathogenesis, as it attenuates the expression of the PU.1 proto-oncogene, a well-known regulator of differentiation in B cells [43]. Moreover, strong miR-155-5p expression was observed in CLL proliferation centers [44]. Nonetheless, the prognostic potential of miR-155-5p expression in CLL has not been studied so far.

To the best of our knowledge, this is the first research study showing that miR-155-5p expression may possess diagnostic value regarding CLL, as miR-155-5p levels are significantly higher in PBMCs of CLL patients, compared to PBMCs of nonleukemic controls. On the other hand, no significant associations were found between miR-155-5p overexpression and CLL patients' clinicopathological features, including Binet stage, Rai stage, risk groups, serum LDH concentration, and cell apoptotic index. However, based on previous results according to which there is a 


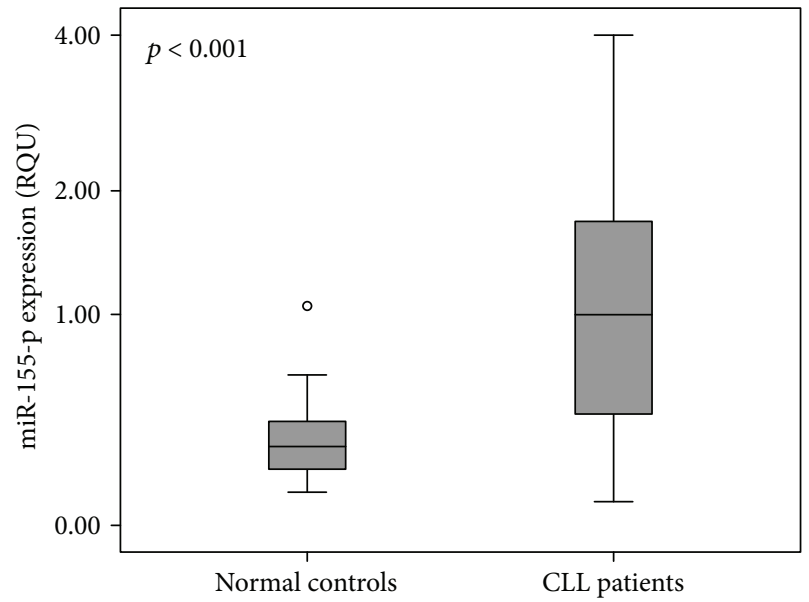

(a)

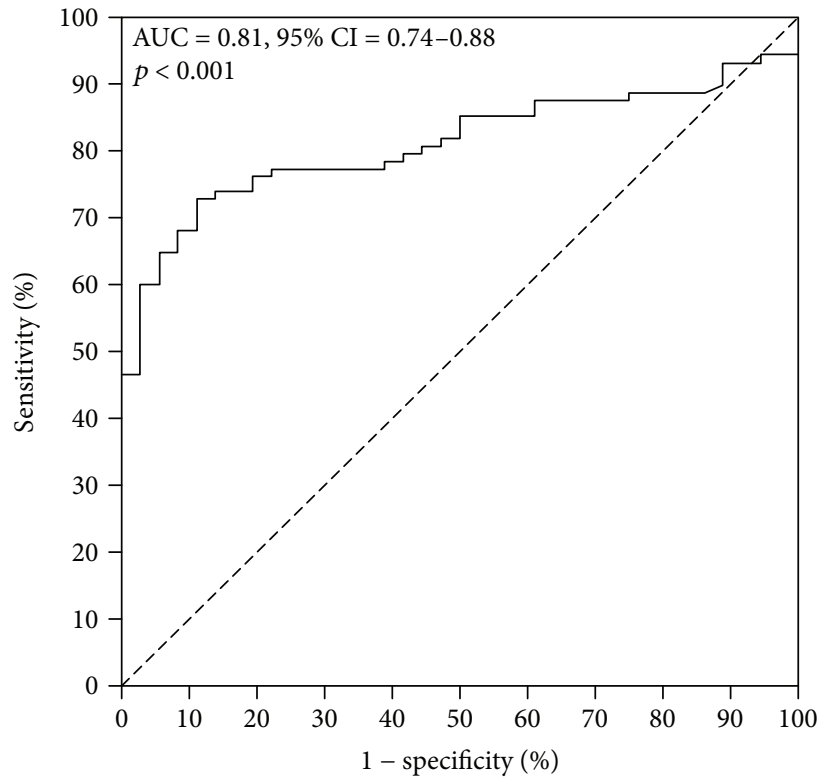

(b)

FIGURE 1: Significant differences are observed in miR-155-5p expression between CLL patients and nonleukemic individuals, thus allowing the discrimination of CLL patients from normal population. (a) Boxplots show the miR-155-5p expression levels in nonleukemic population and CLL patients. The line bars indicate the median value (50th percentile) for each patient cohort, the bottom and top of each box represent the 25th and 75th percentiles, respectively, and the whiskers extend to 1.5 times the height of each box. The $p$ value was calculated using the Mann-Whitney $U$ test. (b) The ROC curve suggests that miR-155-5p expression could be used as a surrogate diagnostic biomarker in CLL.

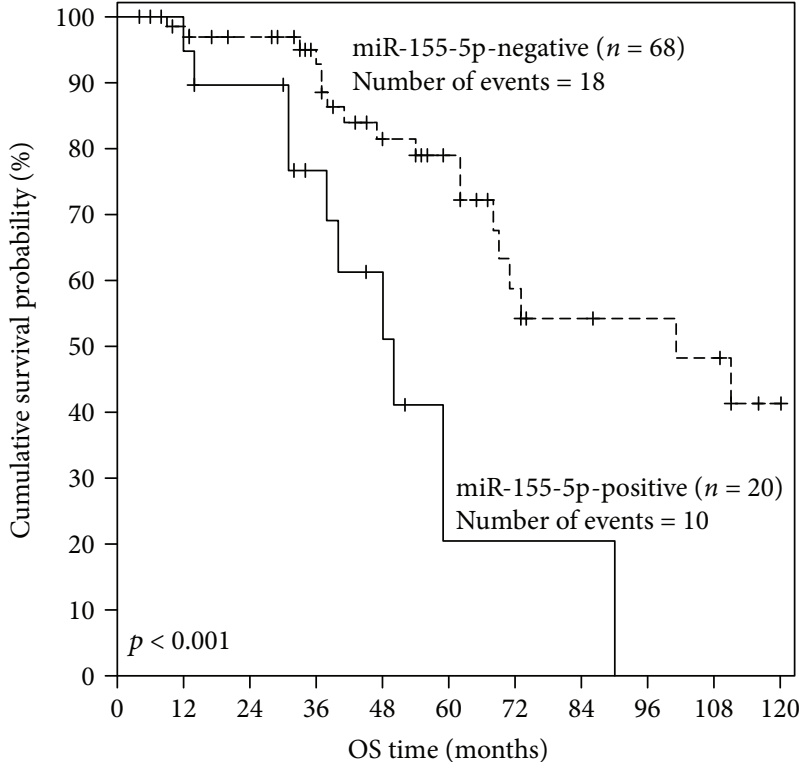

FIgURE 2: Kaplan-Meier OS curves for CLL patients stratified according to miR-155-5p expression in their PBMCs. miR-155-5p expression status is a significant prognostic factor in CLL, as miR155-5p-positive patients have significantly inferior OS, compared to miR-155-5p-negative patients. The $p$ value was calculated using the Mantel-Cox (log-rank) test.

progressive increase in miR-155-5p expression from control cases to patients with monoclonal B cell lymphocytosis and to those with early-stage CLL [45], we decided to examine the putative prognostic value of miR-155-5p expression in CLL.

Although a lot of scientific efforts have been put on the discovery of novel prognostic biomarkers in CLL during the last two decades, only a few of them have led to the establishment of such prognostic biomarkers in the clinics [46]. Staging of the disease, mutations of the $I G H V$ genomic sequences, and loss or mutation of the TP53 gene are probably the most valid prognosticators in CLL $[47,48]$. As it is occasionally rather hard to determine the mutational status of the $I G H V$ gene, several studies sought to identify new promising surrogate markers, such as ZAP70 and CD38 $[49,50]$. Other significant molecular prognostic biomarkers include particular mutations in NOTCH1 [51] and splicing factor $3 \mathrm{~b}$ subunit 1 (SF3B1) genes [52] and chromosomal abnormalities such as deletions of 11q, 13q, 17p, and trisomy $12[53,54]$. In the current study, IGHV mutational status and CD38 expression were verified as significant predictors of prognosis, in contrast to high serum LDH concentration, a typical feature of Richter's transformation that can arise in CLL patients [55]. The mutational status of TP53, SF3B1, and NOTCH1 genes and the aforementioned chromosomal abnormalities were not examined.

Regarding the prognostic value of miR-155-5p expression in CLL, Kaplan-Meier OS analysis revealed significantly lower survival rates for miR-155-5p-positive patients. Moreover, univariate Cox regression analysis indicated that high miR-155-5p expression in PBMCs of CLL patients predicts an increased risk of death. Importantly, the high prognostic significance of miR-155-5p positivity is independent of 
TABLE 2: Cox proportional hazard univariate regression analysis of miR-155-5p expression and clinicopathological variables for the prediction of CLL patients' OS.

\begin{tabular}{|c|c|c|c|c|c|}
\hline Covariate & HR & $95 \% \mathrm{CI}$ & $p$ value & BCa bootstrap (95\% CI) & Bootstrap ( $p$ value) \\
\hline miR-155-5p expression & 1.80 & $1.19-2.72$ & 0.006 & $1.08-3.00$ & 0.007 \\
\hline \multicolumn{6}{|c|}{ miR-155-5p expression status } \\
\hline Negative & 1.00 & & & & \\
\hline Positive & 3.87 & $1.70-8.81$ & 0.001 & $1.76-11.72$ & $<0.001$ \\
\hline Age & 0.98 & $0.94-1.02$ & 0.33 & $0.93-1.02$ & 0.36 \\
\hline Binet stage (ordinal) & 1.98 & $1.3-3.02$ & 0.002 & $1.38-2.97$ & $<0.001$ \\
\hline Rai stage (ordinal) & 1.50 & $1.14-1.98$ & 0.004 & $1.16-2.02$ & 0.002 \\
\hline Risk (ordinal) & 3.01 & $1.52-5.94$ & 0.002 & $1.85-5.29$ & $<0.001$ \\
\hline \multicolumn{6}{|l|}{ Serum LDH concentration } \\
\hline Normal & 1.00 & & & & \\
\hline Elevated & 1.61 & $0.76-3.41$ & 0.22 & $0.74-3.66$ & 0.21 \\
\hline \multicolumn{6}{|l|}{ CD38 expression status } \\
\hline Negative & 1.00 & & & & \\
\hline Positive & 3.57 & $1.68-7.58$ & $<0.001$ & $1.46-13.11$ & $<0.001$ \\
\hline \multicolumn{6}{|l|}{$I G H V$ mutational status } \\
\hline Mutated & 1.00 & & & & \\
\hline Unmutated & 2.78 & $1.18-6.55$ & 0.020 & $1.19-7.99$ & 0.013 \\
\hline
\end{tabular}

CLL: chronic lymphocytic leukemia; OS: overall survival; HR: hazard ratio; CI: confidence interval; BCa: bias-corrected and accelerated; LDH: lactate dehydrogenase; $I G H V$ : immunoglobulin heavy chain variable region.

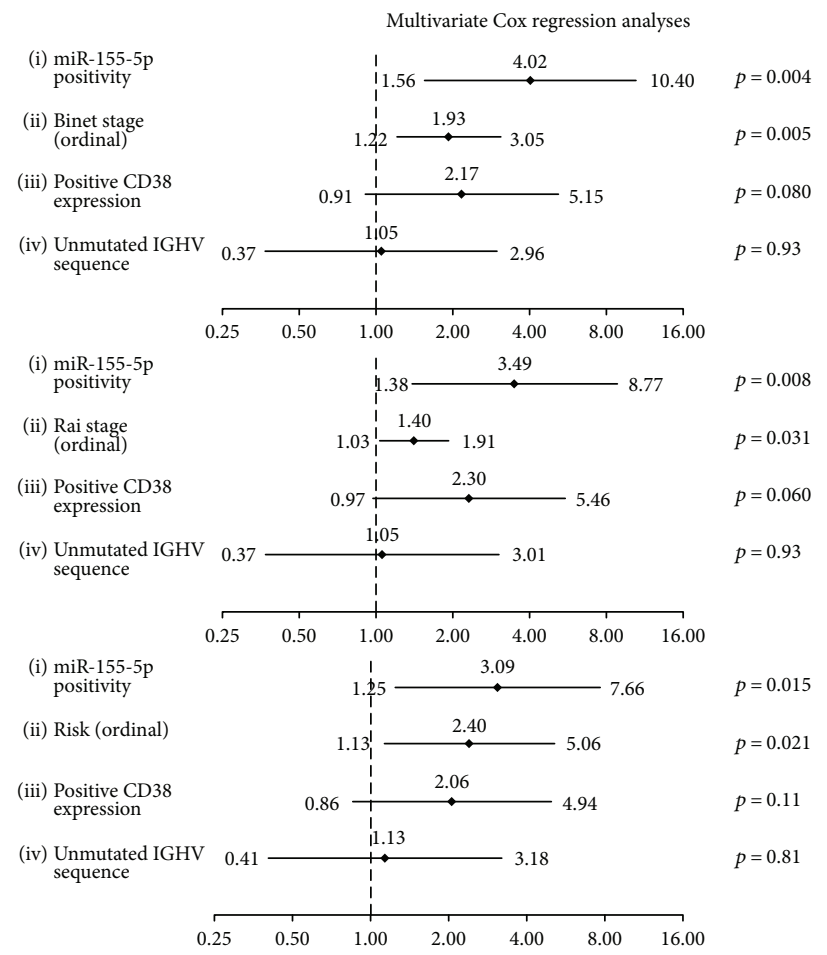

FIgURE 3: Forest plots showing multivariate Cox regression analyses of the effect of miR-155-5p expression status and clinicopathological and biological variables on the OS of CLL patients. The multivariate models were adjusted for the CD38 expression status, presence of IGHV mutations, and either clinical stage (Binet or Rai) or risk group. These two staging systems share common components (enlarged lymph node areas, anemia, and low levels of platelets), and risk classification is based on Rai staging; consequently, these three prognosticators cannot be included in the same multivariate Cox regression model. Diamonds indicate the hazard ratios, and horizontal lines represent the $95 \%$ confidence interval of each hazard ratio. 


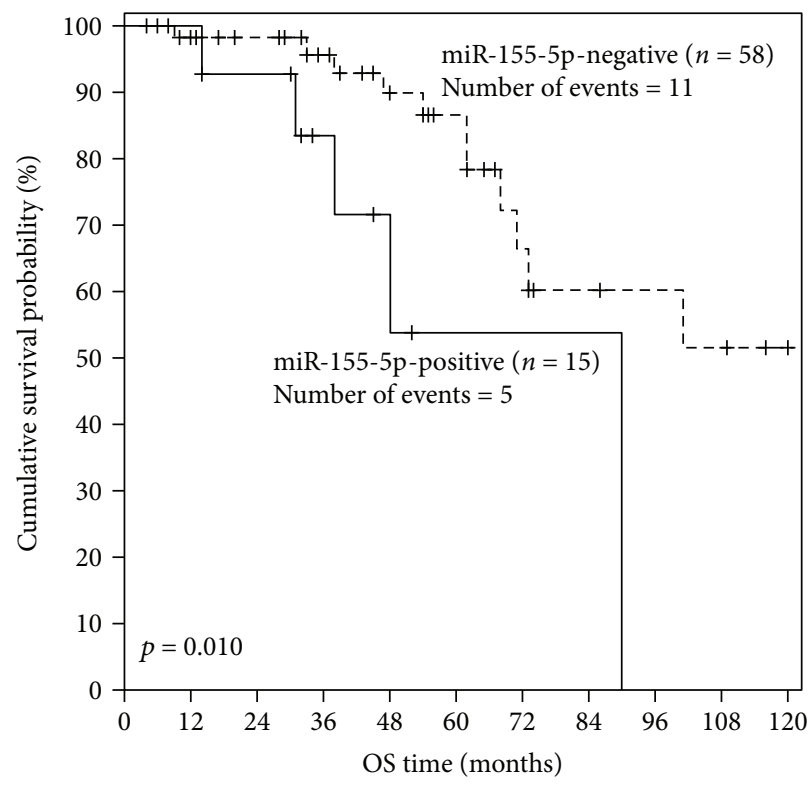

(a)

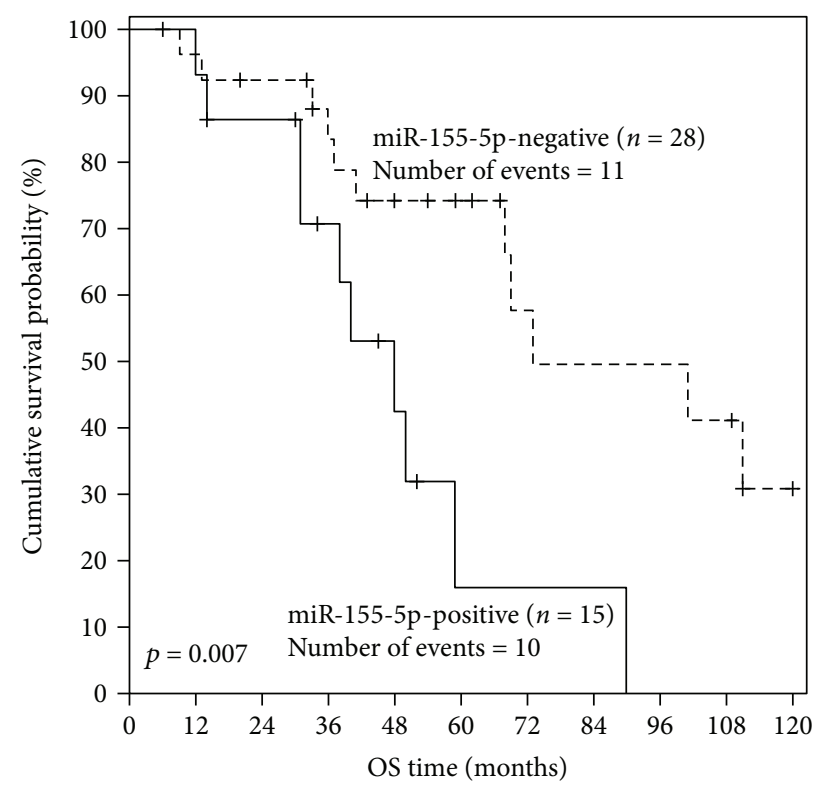

(b)

FIGURE 4: Kaplan-Meier curves for the OS of subgroups of CLL patients with different prognoses. miR-155-5p positivity was shown to have a significant unfavorable prognostic value for CLL patients with (a) negative CD38 expression ( $\leq 30 \%)$ and/or (b) unmutated $I G H V$ genomic sequence.

clinical staging and other prognostic markers, including $I G H V$ mutational status and CD38 expression. Interestingly, according to the developed multivariate Cox regression models, the prognostic significance of miR-155-5p expression status is independent of CD38 expression, unmutated $I G H V$, and the clinical stage (Binet or Rai) or risk category, which represent some of the most valid prognostic markers in CLL. There is no doubt that the prognostic value of miR155-5p expression needs to be further validated in larger and independent cohorts of CLL patients. However, the bootstrapping that was performed in Cox regression strengthens the hypothesis that miR-155-5p overexpression is a promising molecular biomarker of unfavorable prognosis in CLL. Therefore, a panel of molecular prognostic biomarkers that have recently emerged-e.g., the human leukocyte antigen (HLA-G) [56], high histone deacetylase (HDAC) activity [57], mRNA expression of kallikrein-related peptidase 14 (KLK14) [58] and BCL2-like 12 (BCL2L12) [59], circulating miR-150 [60], SRY-box 1 (SOX1) [61], and serum soluble transmembrane activator calcium modulator and cyclophilin ligand interactor (TACI) [62]-could prove even more promising, provided that it integrates miR-155-5p expression as a new component. In support of this suggestion, it should be added that accumulation of several unfavorable prognostic markers has been proven to deteriorate prognosis in CLL [63]. Moreover, it would be tempting to examine the combinatorial value of all these previously suggested prognostic biomarkers besides miR-155-5p expression in a large multicenter study, in order to generate a prognostic score in CLL, like the one that has recently been proposed by the German Chronic Lymphocytic Leukemia Study Group [64].
In conclusion, we developed a cost-effective, sensitive, and accurate SYBR Green-based qPCR technique for the quantification of miR-155-5p levels in PBMCs and investigated its putative discriminatory and prognostic value in CLL. It should be added that the establishment of an optimal cut-off point and the subsequent categorization of miR-155-5p expression values as positive or negative alleviate the need for utmost sensitivity that could be offered by a ready-to-use qPCR assay using TaqMan probes, rendering such commercially available assays less cost-effective [65]. Our study showed that high miR-155-5p expression represents a novel, potential molecular biomarker of unfavorable prognosis in CLL, meriting further investigation in a large cohort of CLL patients. Ideally, miR-155-5p expression status could be combined with surrogate prognostic biomarkers in a multiparametric prognostic index with regard to this hematological malignancy, as the high prognostic significance of miR-155-5p overexpression is independent of clinical staging and other already established biomarkers (IGHV mutational status and CD38 expression) that are used to predict CLL patients' outcome.

\section{Conflicts of Interest}

The authors declare that they have no competing interests.

\section{Authors' Contributions}

Sotirios G. Papageorgiou and Christos K. Kontos contributed equally to this work. 


\section{Supplementary Materials}

Supplementary materials and methods. (Supplementary Materials)

\section{References}

[1] N. Chiorazzi, K. R. Rai, and M. Ferrarini, "Chronic lymphocytic leukemia," The New England Journal of Medicine, vol. 352, no. 8, pp. 804-815, 2005.

[2] G. D'Arena, N. Di Renzo, M. Brugiatelli, M. L. Vigliotti, and M. J. Keating, "Biological and clinical heterogeneity of B-cell chronic lymphocytic leukemia," Leukemia \& Lymphoma, vol. 44, no. 2, pp. 223-228, 2003.

[3] B. Eichhorst, M. Dreyling, T. Robak, E. Montserrat, M. Hallek, and E. G. W. Group, "Chronic lymphocytic leukemia: ESMO Clinical Practice Guidelines for diagnosis, treatment and follow-up," Annals of Oncology, vol. 22, Supplement 6, pp. vi50-vi54, 2011.

[4] K. R. Rai, A. Sawitsky, E. P. Cronkite, A. D. Chanana, R. N. Levy, and B. S. Pasternack, "Clinical staging of chronic lymphocytic leukemia," Blood, vol. 46, no. 2, pp. 219-234, 1975.

[5] J. L. Binet, A. Auquier, G. Dighiero et al., "A new prognostic classification of chronic lymphocytic leukemia derived from a multivariate survival analysis," Cancer, vol. 48, no. 1, pp. 198-206, 1981.

[6] M. Hallek, B. D. Cheson, D. Catovsky et al., "Guidelines for the diagnosis and treatment of chronic lymphocytic leukemia: a report from the International Workshop on Chronic Lymphocytic Leukemia updating the National Cancer InstituteWorking Group 1996 guidelines," Blood, vol. 111, no. 12, pp. 5446-5456, 2008.

[7] C. Moreno and E. Montserrat, "New prognostic markers in chronic lymphocytic leukemia," Blood Reviews, vol. 22, no. 4, pp. 211-219, 2008.

[8] M. Crespo, F. Bosch, N. Villamor et al., "ZAP-70 expression as a surrogate for immunoglobulin-variable-region mutations in chronic lymphocytic leukemia," The New England Journal of Medicine, vol. 348, no. 18, pp. 1764-1775, 2003.

[9] T. J. Hamblin, J. A. Orchard, R. E. Ibbotson et al., "CD38 expression and immunoglobulin variable region mutations are independent prognostic variables in chronic lymphocytic leukemia, but CD38 expression may vary during the course of the disease," Blood, vol. 99, no. 3, pp. 1023-1029, 2002.

[10] T. L. Parker and M. P. Strout, "Chronic lymphocytic leukemia: prognostic factors and impact on treatment," Discovery Medicine, vol. 11, no. 57, pp. 115-123, 2011.

[11] M. A. Valencia-Sanchez, J. Liu, G. J. Hannon, and R. Parker, "Control of translation and mRNA degradation by miRNAs and siRNAs," Genes \& Development, vol. 20, no. 5, pp. 515524, 2006.

[12] A. Esquela-Kerscher and F. J. Slack, "Oncomirs - microRNAs with a role in cancer," Nature Reviews Cancer, vol. 6, no. 4, pp. 259-269, 2006.

[13] V. Balatti, Y. Pekarky, and C. M. Croce, "Role of microRNA in chronic lymphocytic leukemia onset and progression," Journal of Hematology \& Oncology, vol. 55, no. 6, pp. 1227-1239, 2015.

[14] H. Mirzaei, S. Fathullahzadeh, R. Khanmohammadi et al., "State of the art in microRNA as diagnostic and therapeutic biomarkers in chronic lymphocytic leukemia," Journal of Cellular Physiology, vol. 233, no. 2, pp. 888-900, 2017.
[15] I. Faraoni, F. R. Antonetti, J. Cardone, and E. Bonmassar, "miR-155 gene: a typical multifunctional microRNA," Biochimica et Biophysica Acta (BBA) - Molecular Basis of Disease, vol. 1792, no. 6, pp. 497-505, 2009.

[16] D. Yang, J. Wang, M. Xiao, T. Zhou, and X. Shi, "Role of mir-155 in controlling HIF- $1 \alpha$ level and promoting endothelial cell maturation," Scientific Reports, vol. 6, article 35316, no. 1, 2016.

[17] U. Bruning, L. Cerone, Z. Neufeld et al., "MicroRNA-155 promotes resolution of hypoxia-inducible factor $1 \alpha$ activity during prolonged hypoxia," Molecular and Cellular Biology, vol. 31, no. 19, pp. 4087-4096, 2011.

[18] J. M. Brown and W. R. Wilson, "Exploiting tumour hypoxia in cancer treatment," Nature Reviews Cancer, vol. 4, no. 6, pp. 437-447, 2004.

[19] V. Mucaj, J. E. Shay, and M. C. Simon, "Effects of hypoxia and HIFs on cancer metabolism," International Journal of Hematology, vol. 95, no. 5, pp. 464-470, 2012.

[20] E. Tili, C. M. Croce, and J. J. Michaille, " $m i R-155$ : on the crosstalk between inflammation and cancer," International Reviews of Immunology, vol. 28, no. 5, pp. 264-284, 2009.

[21] E. Tili, J. J. Michaille, and C. M. Croce, "MicroRNAs play a central role in molecular dysfunctions linking inflammation with cancer," Immunological Reviews, vol. 253, no. 1, pp. 167-184, 2013.

[22] E. Vigorito, S. Kohlhaas, D. Lu, and R. Leyland, "miR-155: an ancient regulator of the immune system," Immunological Reviews, vol. 253, no. 1, pp. 146-157, 2013.

[23] C. H. Yeh, R. Moles, and C. Nicot, "Clinical significance of microRNAs in chronic and acute human leukemia," Molecular Cancer, vol. 15, no. 1, p. 37, 2016.

[24] S. Costinean, N. Zanesi, Y. Pekarsky et al., "Pre-B cell proliferation and lymphoblastic leukemia/high-grade lymphoma in E $\mu$-miR155 transgenic mice," Proceedings of the National Academy of Sciences of the United States of America, vol. 103, no. 18, pp. 7024-7029, 2006.

[25] T. S. Elton, H. Selemon, S. M. Elton, and N. L. Parinandi, "Regulation of the MIR155 host gene in physiological and pathological processes," Gene, vol. 532, no. 1, pp. 1-12, 2013.

[26] C. K. Kontos, S. G. Papageorgiou, M. A. Diamantopoulos et al., "mRNA overexpression of the hypoxia inducible factor 1 alpha subunit gene (HIF1A): an independent predictor of poor overall survival in chronic lymphocytic leukemia," Leukemia Research, vol. 53, pp. 65-73, 2017.

[27] M. A. Diamantopoulos, C. K. Kontos, D. Kerimis, I. N. Papadopoulos, and A. Scorilas, "Upregulated miR-16 expression is an independent indicator of relapse and poor overall survival of colorectal adenocarcinoma patients," Clinical Chemistry and Laboratory Medicine, vol. 55, no. 5, pp. 737-747, 2017.

[28] K. J. Livak and T. D. Schmittgen, "Analysis of relative gene expression data using real-time quantitative PCR and the $2^{-\Delta \Delta C}$ Tethod," Methods, vol. 25, no. 4, pp. 402-408, 2001.

[29] T. D. Schmittgen and K. J. Livak, "Analyzing real-time PCR data by the comparative $\mathrm{C}_{\mathrm{T}}$ method," Nature Protocols, vol. 3, no. 6, pp. 1101-1108, 2008.

[30] R. L. Camp, M. Dolled-Filhart, and D. L. Rimm, "X-tile: a new bio-informatics tool for biomarker assessment and outcomebased cut-point optimization," Clinical Cancer Research, vol. 10, no. 21, pp. 7252-7259, 2004. 
[31] D. Vasilatou, S. Papageorgiou, V. Pappa, E. Papageorgiou, and J. Dervenoulas, "The role of microRNAs in normal and malignant hematopoiesis," European Journal of Haematology, vol. 84, no. 1, pp. 1-16, 2010.

[32] C. K. Kontos, D. Vasilatou, S. G. Papageorgiou, and A. Scorilas, "Translation regulation by microRNAs in acute leukemia," in Reviews in Cell Biology and Molecular Medicine, Wiley-VCH Verlag GmbH \& Co. KGaA, 2006.

[33] M. Fabbri, C. M. Croce, and G. A. Calin, "MicroRNAs in the ontogeny of leukemias and lymphomas," Leukemia \& Lymphoma, vol. 50, no. 2, pp. 160-170, 2009.

[34] T. H. Thai, D. P. Calado, S. Casola et al., "Regulation of the germinal center response by microRNA-155," Science, vol. 316, no. 5824, pp. 604-608, 2007.

[35] A. Rodriguez, E. Vigorito, S. Clare et al., "Requirement of bic/microRNA-155 for normal immune function," Science, vol. 316, no. 5824, pp. 608-611, 2007.

[36] N. Ling, J. Gu, Z. Lei et al., "microRNA-155 regulates cell proliferation and invasion by targeting FOXO3a in glioma," Oncology Reports, vol. 30, no. 5, pp. 2111-2118, 2013.

[37] K. Miyamoto, K. Y. Araki, K. Naka et al., "Foxo3a is essential for maintenance of the hematopoietic stem cell pool," Cell Stem Cell, vol. 1, no. 1, pp. 101-112, 2007.

[38] I. Faraoni, S. Laterza, D. Ardiri, C. Ciardi, F. Fazi, and F. Lo-Coco, "MiR-424 and miR-155 deregulated expression in cytogenetically normal acute myeloid leukaemia: correlation with NPM1 and FLT3 mutation status," Journal of Hematology \& Oncology, vol. 5, no. 1, p. 26, 2012.

[39] R. Garzon, S. Volinia, C. G. Liu et al., "MicroRNA signatures associated with cytogenetics and prognosis in acute myeloid leukemia," Blood, vol. 111, no. 6, pp. 3183-3189, 2008.

[40] M. Jongen-Lavrencic, S. M. Sun, M. K. Dijkstra, P. J. Valk, and B. Lowenberg, "MicroRNA expression profiling in relation to the genetic heterogeneity of acute myeloid leukemia," Blood, vol. 111, no. 10, pp. 5078-5085, 2008.

[41] S. P. Whitman, K. Maharry, M. D. Radmacher et al., "FLT3 internal tandem duplication associates with adverse outcome and gene- and microRNA-expression signatures in patients 60 years of age or older with primary cytogenetically normal acute myeloid leukemia: a Cancer and Leukemia Group B study," Blood, vol. 116, no. 18, pp. 3622-3626, 2010.

[42] R. M. O'Connell, D. S. Rao, A. A. Chaudhuri et al., "Sustained expression of microRNA-155 in hematopoietic stem cells causes a myeloproliferative disorder," The Journal of Experimental Medicine, vol. 205, no. 3, pp. 585-594, 2008.

[43] K. Vargova, M. Pesta, P. Obrtlikova et al., "MiR-155/miR-150 network regulates progression through the disease phases of chronic lymphocytic leukemia," Blood Cancer Journal, vol. 7, no. 7, article e585, 2017.

[44] K. Szurian, I. Csala, V. Piurko, L. Deak, A. Matolcsy, and L. Reiniger, "Quantitative miR analysis in chronic lymphocytic leukaemia/small lymphocytic lymphoma - proliferation centres are characterized by high miR-92a and miR-155 and low miR-150 expression," Leukemia Research, vol. 58, pp. 39-42, 2017.

[45] F. M. Furtado, P. S. Scheucher, B. A. Santana et al., "Comparison of microRNA expression in high-count monoclonal B-cell lymphocytosis and Binet A chronic lymphocytic leukemia," Revista Brasileira de Hematologia e Hemoterapia, vol. 39, no. 3, pp. 237-243, 2017.
[46] S. Malek, "Molecular biomarkers in chronic lymphocytic leukemia," Advances in Experimental Medicine and Biology, vol. 792, pp. 193-214, 2013.

[47] R. N. Damle, T. Wasil, F. Fais et al., "Ig V gene mutation status and CD38 expression as novel prognostic indicators in chronic lymphocytic leukemia," Blood, vol. 94, no. 6, pp. 1840-1847, 1999.

[48] T. Zenz, A. Krober, K. Scherer et al., "Monoallelic TP53 inactivation is associated with poor prognosis in chronic lymphocytic leukemia: results from a detailed genetic characterization with long-term follow-up," Blood, vol. 112, no. 8, pp. 3322-3329, 2008.

[49] M. I. Del Principe, G. Del Poeta, F. Buccisano et al., "Clinical significance of ZAP-70 protein expression in B-cell chronic lymphocytic leukemia," Blood, vol. 108, no. 3, pp. 853-861, 2006.

[50] G. Del Poeta, L. Maurillo, A. Venditti et al., "Clinical significance of CD38 expression in chronic lymphocytic leukemia," Blood, vol. 98, no. 9, pp. 2633-2639, 2001.

[51] D. Rossi, S. Rasi, G. Fabbri et al., "Mutations of NOTCH1 are an independent predictor of survival in chronic lymphocytic leukemia," Blood, vol. 119, no. 2, pp. 521-529, 2012.

[52] Y. Wan and C. J. Wu, "SF3B1 mutations in chronic lymphocytic leukemia," Blood, vol. 121, no. 23, pp. 4627-4634, 2013.

[53] A. Puiggros, G. Blanco, and B. Espinet, "Genetic abnormalities in chronic lymphocytic leukemia: where we are and where we go," BioMed Research International, vol. 2014, Article ID 435983, 13 pages, 2014.

[54] L. Ripolles, M. Ortega, F. Ortuno et al., "Genetic abnormalities and clinical outcome in chronic lymphocytic leukemia," Cancer Genetics and Cytogenetics, vol. 171, no. 1, pp. 57-64, 2006.

[55] R. Swords, J. Bruzzi, and F. Giles, "Recent advances in the diagnosis and therapy of Richter's syndrome," Medical Oncology, vol. 24, no. 1, pp. 17-32, 2007.

[56] M. A. Attia, N. A. Nosair, A. Gawally, G. Elnagar, and E. M. Elshafey, "HLA-G expression as a prognostic indicator in B-cell chronic lymphocytic leukemia," Acta Haematologica, vol. 132, no. 1, pp. 53-58, 2014.

[57] M. Van Damme, E. Crompot, N. Meuleman et al., "Global histone deacetylase enzymatic activity is an independent prognostic marker associated with a shorter overall survival in chronic lymphocytic leukemia patients," Epigenetics, vol. 9, no. 10, pp. 1374-1381, 2014.

[58] C. K. Kontos, P. G. Adamopoulos, S. G. Papageorgiou, V. Pappa, and A. Scorilas, "mRNA overexpression of kallikrein-related peptidase 14 (KLK14) is an independent predictor of poor overall survival in chronic lymphocytic leukemia patients," Clinical Chemistry and Laboratory Medicine, vol. 54, no. 2, pp. 315-324, 2016.

[59] S. G. Papageorgiou, C. K. Kontos, V. Pappa et al., “The novel member of the BCL2 gene family, BCL2L12, is substantially elevated in chronic lymphocytic leukemia patients, supporting its value as a significant biomarker," The Oncologist, vol. 16, no. 9, pp. 1280-1291, 2011.

[60] B. Stamatopoulos, M. Van Damme, E. Crompot et al., "Opposite prognostic significance of cellular and serum circulating microRNA-150 in patients with chronic lymphocytic leukemia," Molecular Medicine, vol. 21, pp. 123-133, 2015.

[61] H. C. Lai, Y. C. Wang, M. H. Yu et al., "DNA methylation as a biomarker for the detection of hidden carcinoma in 
endometrial atypical hyperplasia," Gynecologic Oncology, vol. 135, no. 3, pp. 552-559, 2014.

[62] M. C. Kyrtsonis, K. Sarris, E. Koulieris et al., "Serum soluble TACI, a BLyS receptor, is a powerful prognostic marker of outcome in chronic lymphocytic leukemia," BioMed Research International, vol. 2014, Article ID 159632, 5 pages, 2014.

[63] M. S. Truger, S. Jeromin, S. Weissmann et al., "Accumulation of adverse prognostic markers worsens prognosis in chronic lymphocytic leukaemia," British Journal of Haematology, vol. 168, no. 1, pp. 153-156, 2015.

[64] N. Pflug, J. Bahlo, T. D. Shanafelt et al., "Development of a comprehensive prognostic index for patients with chronic lymphocytic leukemia," Blood, vol. 124, no. 1, pp. 49-62, 2014.

[65] S. M. Rapti, C. K. Kontos, I. N. Papadopoulos, and A. Scorilas, "Enhanced miR-182 transcription is a predictor of poor overall survival in colorectal adenocarcinoma patients," Clinical Chemistry and Laboratory Medicine, vol. 52, no. 8, pp. 1217-1227, 2014. 


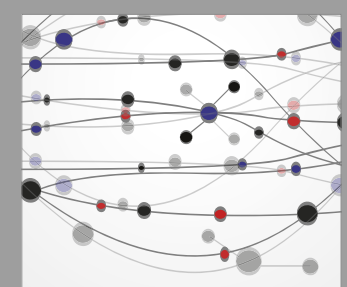

The Scientific World Journal
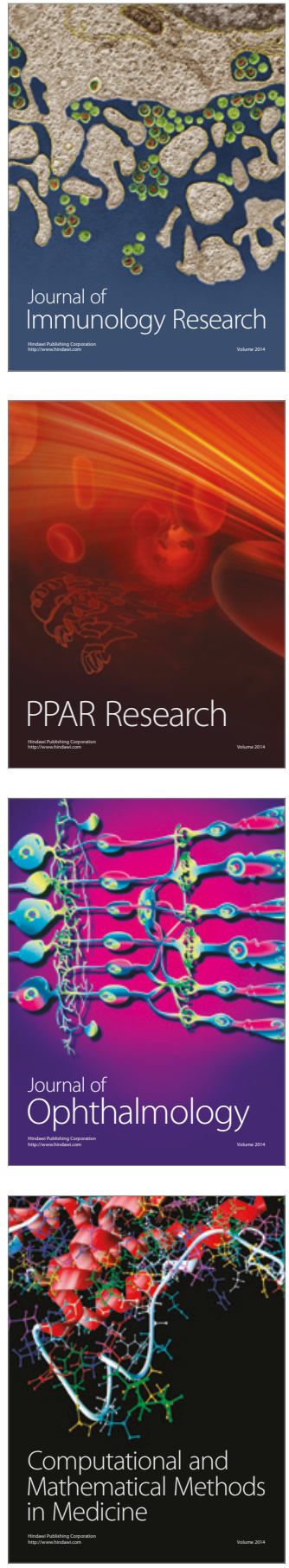

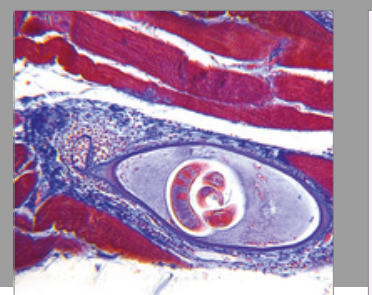

Gastroenterology Research and Practice
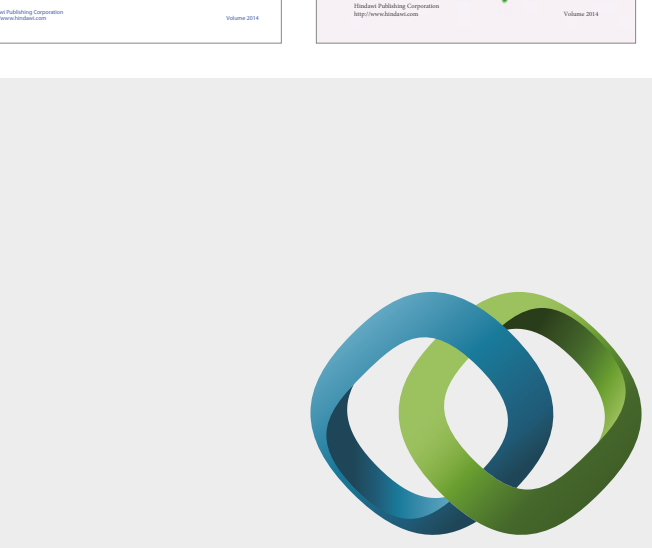

\section{Hindawi}

Submit your manuscripts at

https://www.hindawi.com
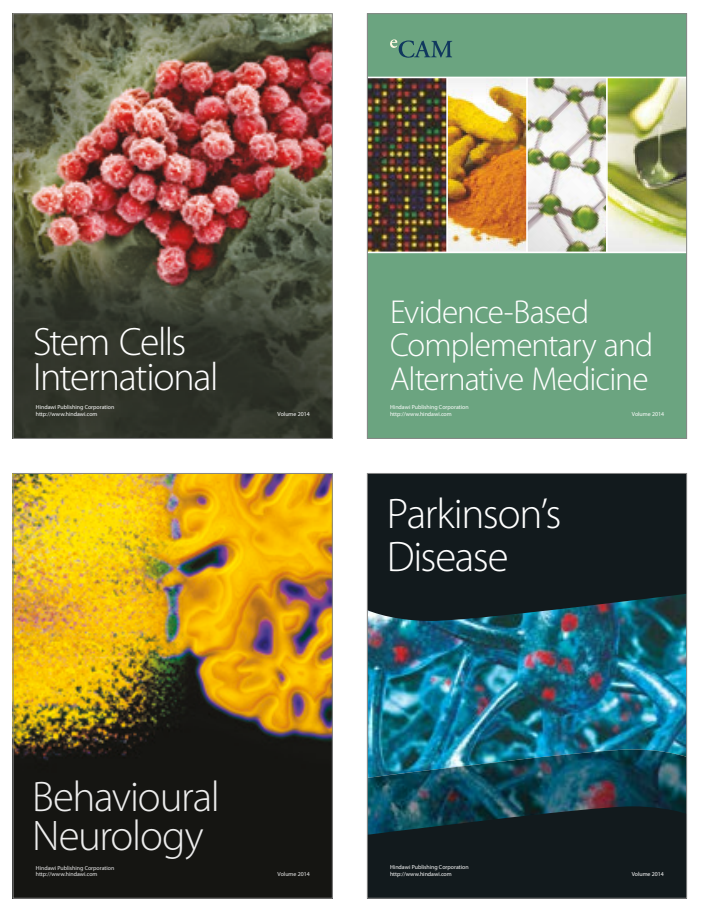
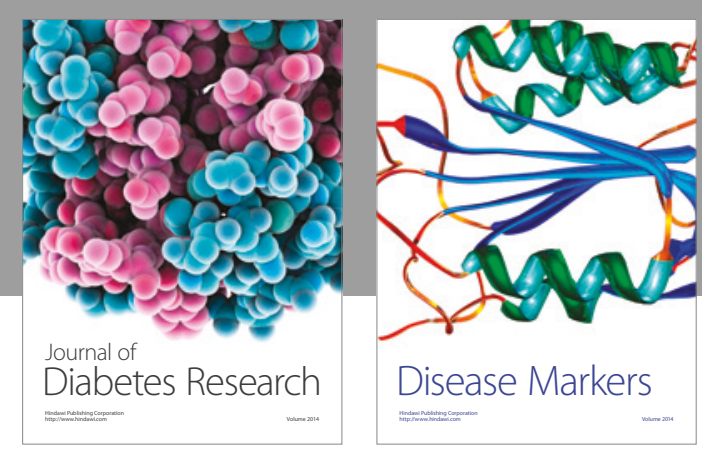

Disease Markers
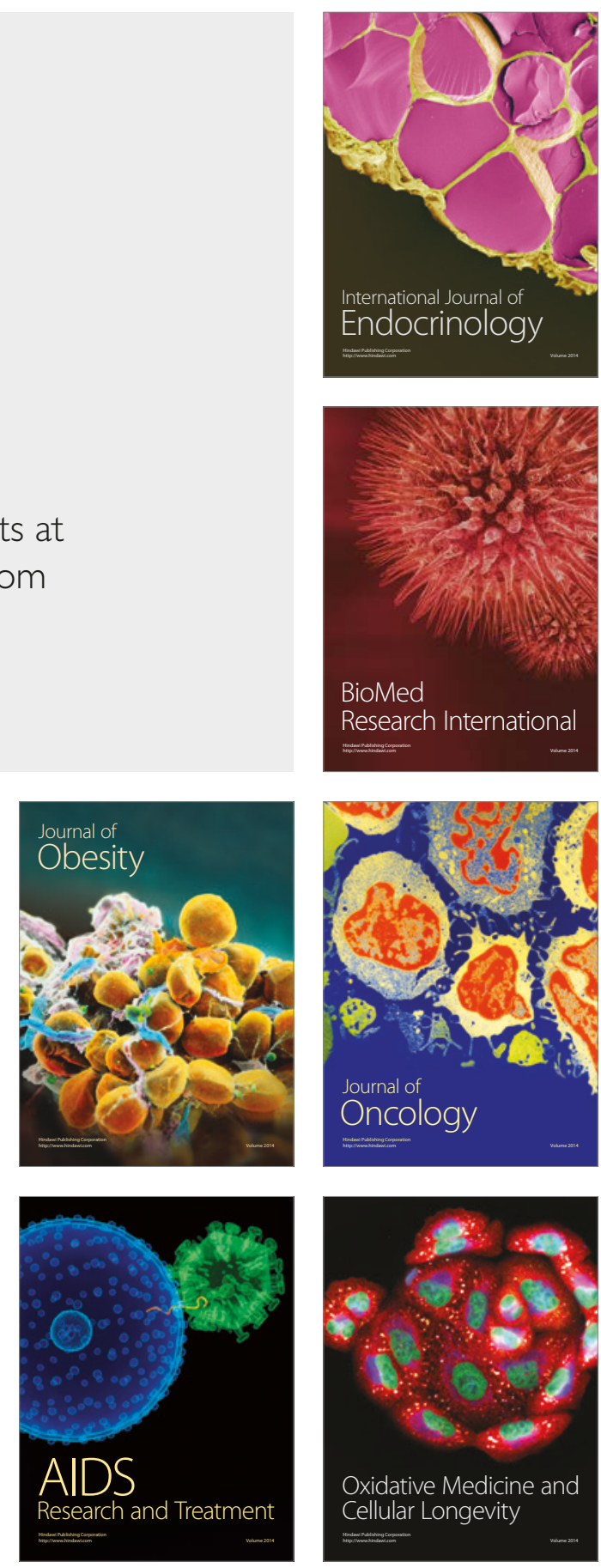